\title{
Effects of Compliance in Pedundulatory Locomotion over Granular Substrates
}

\author{
Michael Sfakiotakis, Avgousta Chatzidaki, Theodoros Evdaimon, Asimina Kazakidi, and Dimitris P. Tsakiris
}

\begin{abstract}
The present paper investigates the effect of compliance on the locomotion of a biologically-inspired soft-body pedundulatory robotic system, employing lateral undulations of its elongated body, which are augmented by the oscillation of sets of lateral appendages (parapodia), to propel itself on unstructured granular substrates. We explore control strategy alternatives for the robot to generate two different locomotor gaits by employing direct or retrograde lateral body waves, combined with appropriately coordinated parapodial motion (pedundulatory modes). Computational models of this class of robots have been devised, which demonstrate the effects of joint compliance on gait generation and on the characteristics of robot propulsion. A new three-segment soft-body robotic prototype has been developed, whose body was fabricated by molding polyurethane elastomers, and was tested extensively on an experimental sandbox, on various formations of the granular substrate, to compare the performance of stiff and compliant joints. Body and joint compliance were found to enhance the adaptability of the robot to environmental irregularities, however they may deteriorate the proper formation of the undulatory body wave, degrading somewhat system performance in terms of the attained velocities.

Index Terms-Biologically Inspired Systems, Robotics, Soft
\end{abstract} Robotics, Undulatory Robotics, Compliance, Sand.

\section{INTRODUCTION}

Robotic locomotion by means of body undulations, has attracted significant interest due to its potential for efficiency and performance in unstructured environments [1][9]. Particularly noteworthy such domains are sand and other granular substrates, where the transitions between states of aggregation of the granular matter, as a result of the movement of robotic systems, may challenge their ability to propel themselves in these environments [10]-[12]. Our previous investigations of undulatory and pedundulatory robotic locomotion on sand, and other compliant substrates, were inspired by the polychaete annelid marine worms and the centipede terrestrial arthropods (Fig. 1), and demonstrated (a)

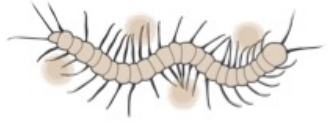

(b)

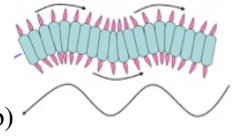

Fig. 1: The coordination between parapodial oscillations and body undulations in (a) centipedes and (b) polychaete annelids.

This work was supported in part by Greek national funds through the Operational Program "Education and Lifelong Learning" of the National Strategic Reference Framework - Research Funding Program: THALES via the BioLegRob Project (Mis: 379424).

The authors are with the Institute of Computer Science, Foundation for Research and Technology - Hellas (FORTH), Heraklion, Greece. M. Sfakiotakis is also with the Dept. of Electrical Engineering, Technological Educational Institute of Crete, Heraklion, Greece. \{sfakios, avgousta, evdemon, kazakidi, tsakiris\}eics.forth.gr.

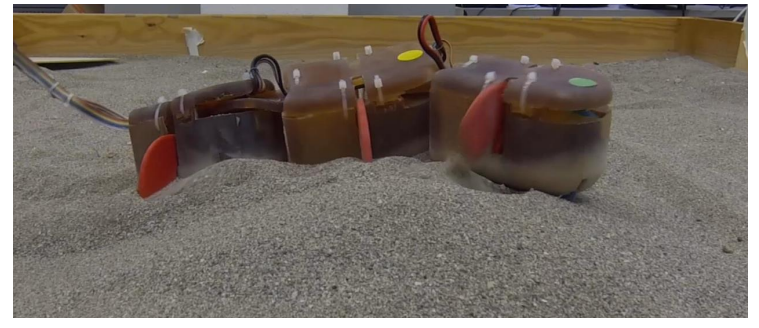

Fig. 2: The Nereis compliant pedundulatory robotic prototype, passively adapting its shape to that of a sand hill.

gait generation, neuromuscular control and the emergence of reactive behaviors for rigid-body such systems [13]-[18]

One important characteristic of living organisms, which is starting to be explored in undulatory robotics, is passive or active compliance of their bodies, which may assist their effortless adaptability to irregular features of the terrain [19] [26]. Along this axis, this paper focuses on the role of passive in-series compliance [19]-[21] on the propulsion characteristics of pedundulatory robotic systems over granular substrates, an issue studied both computationally and experimentally. In particular, we compare the use of rigid and compliant joints in a compliant-body robot (Fig. 2), employing either direct (tail-to-head) or retrograde (head-totail) body undulations, combined with oscillations of paddlelike lateral appendages (named parapodia). These, when appropriately synchronized with the undulations of the body, provide a locomotor advantage over irregular and unstructured terrains. Such a capability may be important for robotic applications in unstructured domains, like robotic endoscopy of the gastrointestinal tract, vascular interventions, search \& rescue operations, and space exploration [27].

The presented work also differs from our previous studies [13]-[18] with regard to the novel design and significantly more challenging fabrication process of the compliant body of the developed Nereis robotic prototype (Fig. 2), an 8-dof robot comprised of three compliant segments interconnected by compliant joints, and a total of six parapodia. The results, reported here, provide evidence that compliance assists the robot to adapt to the irregularities of unstructured granular substrates, and increases the robustness of its implemented behaviors, as well as the safety of its interaction with the environment. However, the compliant body also makes the system heavier, and harder to model, implement and control, and may reduce the propulsive force it is able to generate, and, thus, its attained speed. Moreover, the level of compliance required for a specific task, which, in our case, is being implemented via the selection of the joint material and geometry, may be hard to assess and pre-select. 
In the remainder of the paper, Section II describes the computational model of the compliant-joint robot; Section III outlines the motion control for pedundulatory gaits, while Section IV presents computational studies for the effect of compliance. Section V describes the development of the compliant Nereis robot, and the related experimental setup, with Section VI presenting experimental results on sand. Finally, Section VII discusses further the results of this work.

\section{COMPUTATIONAL MODELLING}

\section{A. Mechanism Model}

A simplified planar mechanical model of the Nereis compliant pedundulatory robot, is shown in Fig. 3. It is composed of a planar serial arrangement of 3 identical links (segments $B_{j}$, where $j=1 . .3$ ) of length $l_{b}$, interconnected by planar rotary joints whose actively-controlled joint angles are denoted by $\phi_{i}, i=1,2$. Three is the minimum number of links that could be implemented in our mechanical model in order to achieve the proposed motion profile; nevertheless, this number is not limiting, and more links may give rise to different behaviours. On each segment, a pair of laterally placed parapodial links of length $l_{p}$ and mass $m_{p}$ is mounted at a distance $b$ from the segment's rear edge. The parapodia (designated as $P_{1} \ldots P_{6}$ in Fig. 3a) are connected to the segment via planar dorsoventrally-oriented single-degree-offreedom rotary joints. When all parapodia are retracted, the mechanism is essentially reduced to an undulatory system. In pedundulatory modes, the overall desired "paddling" action of the parapodia emerges when their up-down movements are combined with body undulations.

Compliant joints: In the Nereis prototype (Section V-A), the body segments are connected by a compliant joint element (a thick polyurethane strip), which can bend both horizontally and vertically, as well as twist. Currently, these effects cannot be fully captured by our planar simulation setup. As a first approximation, horizontal joint compliance is modeled by an additional unactuated 1-dof rotary joint, co-planar with the active body joints, which is located at a distance $l_{e}$ from the actuated body joint, as in Fig. 3b, and equipped with a torsional spring of stiffness $k$. The angular position of this passive joint is denoted as $\alpha_{i}$.

\section{$B$. Interaction with the Environment}

The frictional interaction of the pedundulatory mechanism's $j$ th body segment with the environment is described here by a Coulomb force model, involving the decoupled components (cf. Fig. 3a):

$$
F_{T}^{B j}=-\mu_{T} m_{b} g \operatorname{sgn}\left(v_{T}^{B j}\right), F_{N}^{B j}=-\mu_{N} m_{b} g \operatorname{sgn}\left(v_{N}^{B j}\right),
$$

acting in the tangential and normal direction of the segment motion, where $v_{T}^{B j}$ and $v_{N}^{B j}$ are the respective velocity components, while $m_{b}$ is the segment's mass and $g$ is the constant of gravity. The tangential $\left(\mu_{T}\right)$ and normal $\left(\mu_{N}\right)$ Coulomb friction coefficients are of key significance for the development of the investigated locomotion modes.

A similar approach is adopted for the parapodia, where the Coulomb-like model:

$$
F_{T}^{P_{n}}=-f_{T} \operatorname{sgn}\left(v_{T}^{P_{n}}\right) \text { and } F_{N}^{P_{n}}=-f_{N} \operatorname{sgn}\left(v_{N}^{P_{n}}\right),
$$

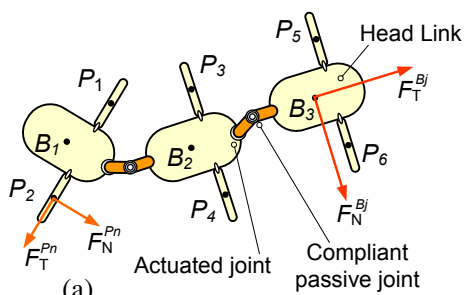

(a)

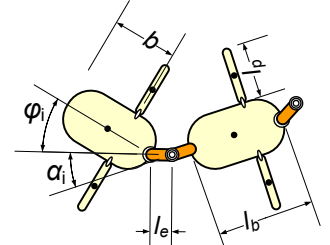

(b)
Fig. 3: (a) Mechanical model of the 3-segment pendundulatory Nereis robot with compliant body joints. (b) Detail of the compliant joint model.

may be used, as a first approximation, to describe the forces generated by parapodium $P_{n}(n=1 \ldots 6)$, when in contact with the locomotion substrate (Fig. 3a). The magnitudes $f_{N}$ and $f_{T}$ of these forces can be determined experimentally.

A computational model of the full body dynamics of the above system, incorporating the mechanical parameters (masses, dimensions, etc.) of the actual robotic prototype, has been implemented in the SIMUUN simulation environment, as described in detail in [16], which is based on the SimMechanics toolbox of Matlab/Simulink.

\section{PEDUNDULATORY GAIT GENERATION}

This Section presents the motion control strategies, which give rise to the pedundulatory modes under investigation.

In purely undulatory mode (i.e., when all parapodia are retracted), locomotion is obtained through the coupling of the mechanism's shape changes to the frictional forces applied through the interaction with the environment. In the present study, the latter are described by the Coulomb friction model (1), where the tangential $\left(\mu_{T}\right)$ and normal $\left(\mu_{N}\right)$ friction coefficients depend on the shaping and material of the links' underside, as well as on the material properties of the substrate (for details, see [15] and refs. therein).

In the pedundulatory modes, body undulations are combined with the periodic activation of the lateral parapodial appendages, which occurs in waves propagating in the same direction as the body undulations. Appropriate coordination is required, in order to ensure the positive contribution of the parapodia in thrust generation during each locomotion cycle. Two main such pedundulatory modes have been developed [13], [14], [18], which are inspired by the locomotion of centipedes (for retrograde wave propagation) and of polychaete marine worms (for direct wave propagation, Fig. 1). Note that our mechanical system (Fig. 3) cannot propel itself by the simple dorso-ventral parapodial movements alone; it is the traveling body wave that effectively positions the parapodia, allowing them to move backwards with respect to the substrate, and to thus impart propulsive forces.

Polychaete mode: For rapid crawling and swimming, the polychaete worms combine tail-to-head body undulations with alternating waves of parapodial activity, coordinated so that the thrust-producing power stroke of each parapodium occurs at the crest of the body wave, while its recovery phase occurs in the trough of the body wave [28], [29]. Assuming full position control of the mechanism's joint angles, the implementation of this gait is based on a tail-to-head body wave of uniform amplitude, by specifying the trajectories of the body joints according to the following equation: 

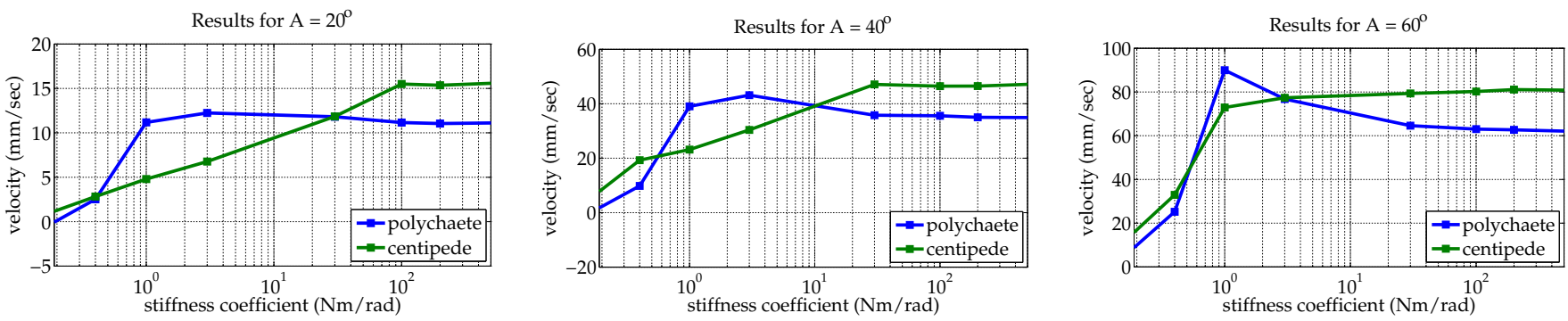

Fig. 4: Simulation results: Average attained velocity of the three-segment robot, as a function of the stiffness coefficient $k$ of the passive rotary joints, for different values of the actuated body joints' oscillation amplitude $A$.

$$
\phi_{i}(t)=A \sin \left(2 \pi f t+(3-i) \varphi_{0}\right)+\psi, i=1,2,
$$

where $A, f$ and $\psi$ denote, respectively, the amplitude, frequency and angular offset of the joint sinusoidal motion, while $\varphi_{0}>0$ is the phase shift between consecutive joints. This is complemented by the appropriately coordinated action of the parapodia described in [13], [14], [18].

Centipede mode: Centipedes combine, for forward locomotion, head-to-tail undulations of their segmented body with leg movements, synchronized so that the legs make contact with the substrate when the corresponding body segment is in the trough of the body wave [30]. The implementation of this gait involves a retrograde (i.e., head-to-tail) body wave of uniform amplitude, attained as:

$$
\phi_{i}(t)=A \sin \left(2 \pi f t+i \varphi_{0}\right)+\psi, i=1,2,
$$

complemented by the appropriately coordinated action of the parapodia, as described in [13], [14], [18].

Pedundulatory turning: Turning motions may be implemented for the two pedundulatory modes in a number of different ways. The simplest one, involves introducing an angular offset $\psi \neq 0$ in the body wave (3),(4), while the parapodia operate in the normal bilateral fashion of the corresponding pedundulatory mode. The path curves in the clockwise or counter-clockwise direction, depending on the sign of $\psi$ and the body wave direction. Other possibilities on pedundulatory turning strategies are discussed in [14], [18].

\section{SIMULATION RESULTS}

Simulations were carried out for the two pedundulatory locomotion modes, using the SIMUUN model of the threelink Nereis prototype with compliant joints (Fig. 3). The system parameters are summarized in Table II. The values of the body friction coefficients $\mu_{N}, \mu_{T}$ were obtained from the experiments presented in Section VI, while those of the parapodial forces $f_{n}, f_{t}$ from prior measurements [18]. The simulation studies presented here focused mainly on the effect of joint compliance, by varying the stiffness coefficient

TABLE I: Model parameters used in the simulation studies.

\begin{tabular}{rrr}
\hline Parameter & Value & Unit \\
\hline Body segment length, $l_{b}$ & 0.10 & {$[\mathrm{~m}]$} \\
Body segment $B_{1}, B_{2}$ mass, $m_{b}$ & 0.410 & {$[\mathrm{~kg}]$} \\
Head body segment $B_{3}$ mass, $m_{b}$ & 0.610 & {$[\mathrm{~kg}]$} \\
Length of parapodia, $l_{p}$ & 0.035 & {$[\mathrm{~m}]$} \\
Mass of parapodia, $m_{p}$ & 0.015 & {$[\mathrm{~kg}]$} \\
Compliant joint length, $l_{e}$ & 0.06 & {$[\mathrm{~m}]$} \\
Distance of parapodia, $b$ & 0.06 & {$[\mathrm{~m}]$} \\
Normal friction force of parapodia, $f_{N}$ & 2.5 & {$[\mathrm{~N}]$} \\
Tangential friction force of parapodia, $f_{T}$ & 0.1 & {$[\mathrm{~N}]$} \\
Tangential friction coefficient of body segments, $\mu_{T}$ & 0.44 & {$[-]$} \\
Normal friction coefficient of body segments, $\mu_{N}$ & 0.5 & {$[-]$} \\
\hline
\end{tabular}

$k$ of the passive joints interconnecting the body segments. For high values of $k$, the system is essentially reduced to a rigid-joint mechanism. In all cases, the phase shift between the oscillations of the two body joints was specified as $\varphi_{0}=120^{\circ}$. Fig. 4 summarizes the simulation results for the average steady-state velocity of the system, as a function $k$ and for different values of the actuated joints' oscillation amplitude $A$. The attained velocities are relatively low, due to the limited number of segments of the system. It can be seen that for both modes, but mostly for centipede locomotion, the performance generally declines as joint compliance is increased (i.e., for small values of $k$ ), irrespectively of the amplitude $A$. On the other hand, the centipede mode attains higher velocities for rigid joints (i.e., for large values of $k$ ). It should be noted that these findings are dependent on the kinematic and tribological parameters of the system.

The graphs in Fig. 5 show the trajectories of the three segments' center of mass, for the two pedundulatory modes, obtained with $A=60^{\circ}$ for $k=3 \mathrm{Nm} / \mathrm{rad}$. It can be seen that the lateral displacements of the middle segment are significantly larger than those of the tail and head segments.

Fig. 6 shows the time course of the passive joint angles $\alpha_{i}$ (cf. Fig. 3), for two different values of the parameter $k$ in polychaete mode (corresponding results can be obtained for centipede mode). The reduction of the stiffness of the com-

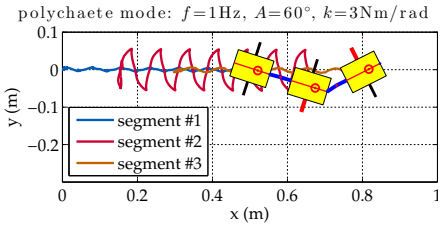

(a) polychaete mode

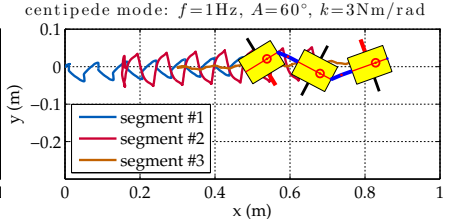

(b) centipede mode
Fig. 5: Simulation results: The trajectories of the 3 segments of the mechanism in the two pedundulatory modes, for $k=3 \mathrm{Nm} / \mathrm{rad}$.

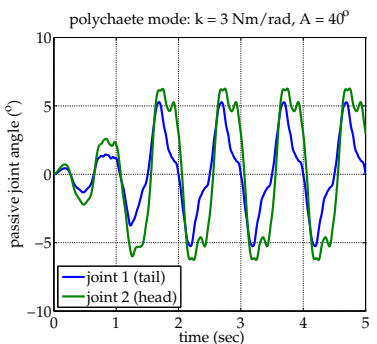

(a) $k=3 \mathrm{Nm} / \mathrm{rad}$

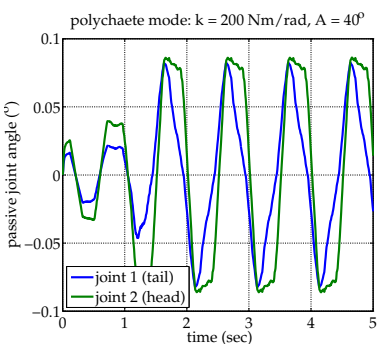

(b) $k=200 \mathrm{Nm} / \mathrm{rad}$
Fig. 6: Simulation results: Time course of the angles $\alpha_{i}$ of the system's passive rotary joints, for two values of the stiffness coefficient $k$, in polychaete mode. 


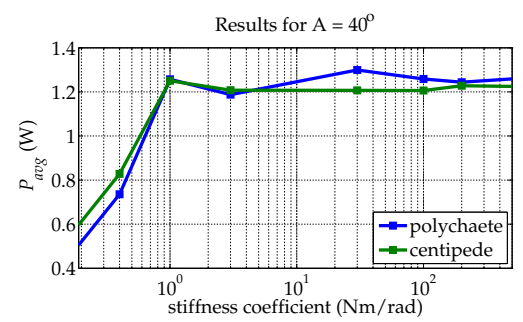

Fig. 7: Simulation results: Average total power expenditure at the actuated body joints of the system, as a function of the stiffness coefficient $k$ of the passive rotary joints.

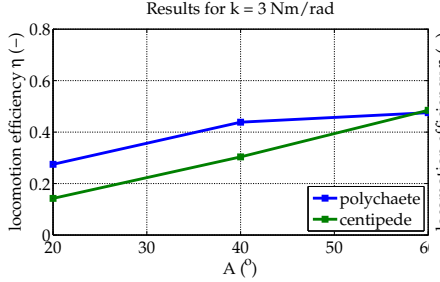

(a) $k=3 \mathrm{Nm} / \mathrm{rad}$

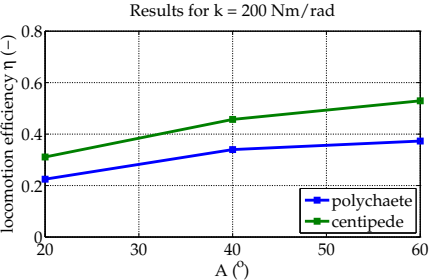

(b) $k=200 \mathrm{Nm} / \mathrm{rad}$
Fig. 8: Simulation results: Locomotion efficiency of the system, as a function of the amplitude $A$, for two stiffness coefficients $k$ of the passive rotary joints.

pliant interconnection elements results in an increase of the passive joints' angular motion span, leading to a distortion of the body undulation pattern, generally associated with a reduction of the attained locomotion speed.

Power requirements were assessed in terms of the average total power expenditure, for the implementation of the body wave over one undulation period $T=1 / f$, calculated as:

$$
P_{a v g}=\frac{1}{T} \int_{t_{0}}^{t_{0}+T} \sum_{i=1}^{2}\left|\tau_{i}(t) \dot{\phi}_{i}(t)\right| d t,
$$

where $t_{0}$ specifies a time instance in the steady-state response of the system, while $\tau_{i}(t)$ and $\dot{\phi}_{i}(t)$ are the torque and angular velocity of the mechanism's $i$ th body joint. The latter are calculated from the dynamics of the system, for each simulation run, by the SimMechanics toolbox. The power contribution from the parapodial actuators are not involved in the calculation of $P_{a v g}$, since the contribution from the body joint actuators is considerably higher. A plot of $P_{a v g}$, as a function of the stiffness coefficient $k$, for $A=40^{\circ}$, is provided in Fig. 7. It can be seen that, for both pedundulatory modes, the power expenditure increases with the joint stiffness.

The locomotion efficiency of the system is calculated as $\eta=M g U / P$, where $M$ is the overall mass of the mechanism, $g$ is the gravitational constant, and $U$ the average steady-state velocity attained by the robot. Our results, summarized in Fig. 8, indicate that, for both locomotion modes, $\eta$ generally increases with the prescribed oscillation amplitude $A$ of the actuated body joints, up to a value of approximately 0.52 .

\section{PROTOTYPE DESIGN \& DEVELOPMENT}

\section{A. Robotic prototype design}

Within the scopes of the current paper, we have developed a new 3-segment Nereis robotic prototype (Fig. 2), with compliant body and joints, and rigid parapodia. The robot weighs $1415 \mathrm{gr}$ and is $380 \mathrm{~mm}$ in length. Each segment is comprised of a compliant outer shell (Fig. 9a, 10a), a

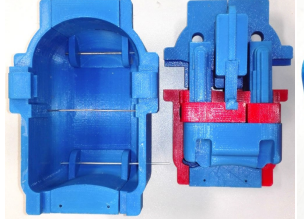

(a)

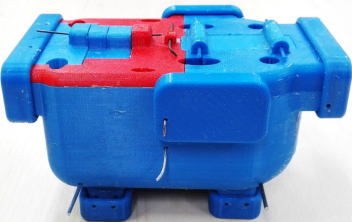

(b)

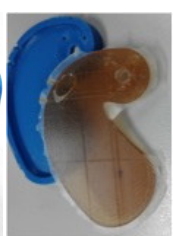

(c)
Fig. 9: 3d-printed casts for fabrication of $(a, b)$ a segment shell and (c) a small compliant parapodium.

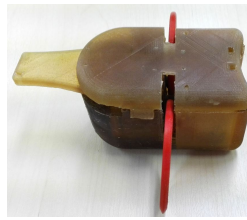

(a)

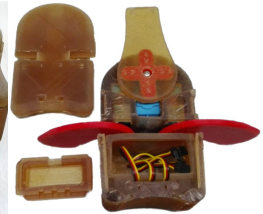

(b)

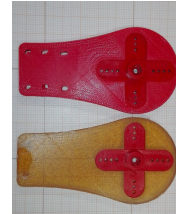

(c)

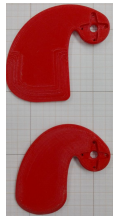

(d)
Fig. 10: Compliant polyurethane-made robot components: (a) a complete segment, (b) a segment with lids removed, (c) rigid (top) and compliant (bottom) joint elements, and (d) large (top) and small (bottom) rigid parapodia.

coupling joint element, the electronics' case, an upper lid of the shell, two lateral parapodia, two waterproof servomotors (Hitec HS-5086WP) that actuate the parapodia, and a third waterproof servomotor (Hitec HS-5646WP), that actuates the segment joint. The development of each component of the prototype was the result of an investigation for the optimal design characteristics of the robot, based on the servomotors' performance specifications and overall dimensions, the joints' stress-strain analysis, and several evaluation tests.

Each segment (Fig. 10a) is $120 \mathrm{~mm} \times 74 \mathrm{~mm} \times 74 \mathrm{~mm}$ in length, width and height, respectively, with a wall thickness of $2.5 \mathrm{~mm}$. The front part of each segment has a semiellipsoidal shape, to facilitate locomotion on sand. The joint elements, interconnecting two successive segments, can greatly influence the performance of the robot (Fig. 10c). Both rigid and compliant such elements were developed, measuring $88 \mathrm{~mm} \times 48 \mathrm{~mm} \times 6.5 \mathrm{~mm}$ in length, maximum width and thickness, respectively. Each segment incorporates two lateral parapodia (Figs. 9c and 10d), driven by dedicated servomotors that move each parapodium independently in a plane perpendicular to the direction of the robot's motion. Various parapodia designs, both rigid and compliant, have been fabricated, with the rigid variants employed in the present study being $70 \mathrm{~mm} \times 40 \mathrm{~mm} \times 5 \mathrm{~mm}$, with a sand immersion depth of $20 \mathrm{~mm}$. Equipped with these parapodia and compliant joints, each segments' weight is $430 \mathrm{gr}$.

\section{B. Robotic prototype fabrication}

The present work investigates the exploitation of compliant materials for the fabrication of the robot's body and joints. This presents considerable technical challenges, as it involves the design of castable elastomeric body parts and their interlocking assembly to form a single unit. The design should allocate appropriate space for the incorporation of rigid components, such as servomotors, electronics and joint gearings, in a way that enhances the overall stiffness of the segment to avoid excessive deformations. The compliant shell of each segment is fabricated in 3D-printed molds (Fig. $9 \mathrm{a}, \mathrm{b})$, the design of which introduces further complications, 


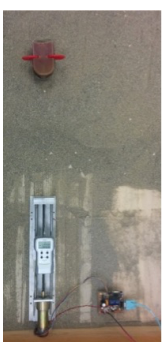

(a) experimental setup

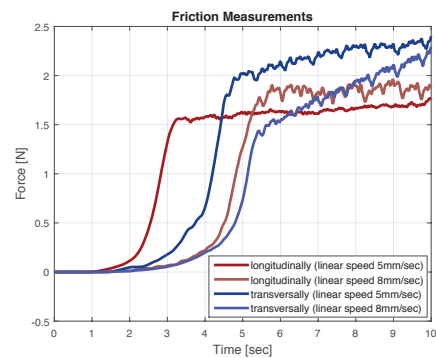

(b) results
Fig. 11: Measurement of the body segment frictional force.

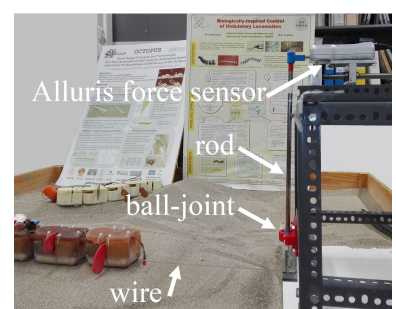

(a) experimental setup

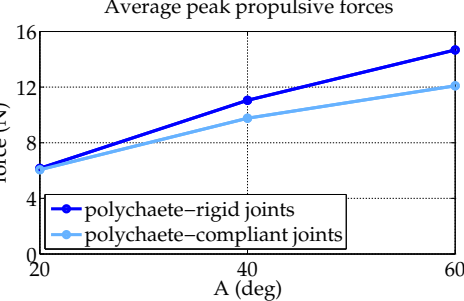

(b) results
Fig. 12: Measurement of the propulsive force of the robot.

due to the multiple parts required to fit exactly together, both internally and externally, in order to achieve the required cast (Fig. 10a,b). Disassembly of the molds reveals the fabricated compliant part; the molds can then be reused for further casting (e.g., Fig. 9c).

The material used for the fabrication of the prototype is a two-component polyurethane (PMC-746, Smooth-On). ABSplus-made molds were fabricated for each compliant component of the robot, to host the polyurethane mixture (Fig. 9). A room-temperature casting procedure is followed, whereby the liquid polyurethane mixture is injected from the top face of the molds, at small quantities at a time, in order to let trapped air escape through purpose-built holes. The pot life of the material is $15 \mathrm{~min}$ and cures overnight into an A60 shore hardness rubber, with $100 \%$ modulus of $220 \mathrm{psi}$, and tensile strength of 700psi. The final mixture results in an amorphous polymer that exhibits viscoelastic behavior.

Bending tests were performed on the compliant joint elements (Fig. 10c-bottom), and the horizontal joint element compliance was estimated as $2.82 \mathrm{Nm} / \mathrm{rad}$.

\section{Robot electronics}

Mounted on the robot's head segment is a Teensy 3.1 platform, based on the ARM Cortex M4 microcontroller, which generates the PWM signals for the RC servos according to the desired motion profiles of the selected locomotion mode. Power to the various electronic components is provided by an on-board $7.4 \mathrm{~V} \mathrm{LiPo} \mathrm{battery} \mathrm{through} \mathrm{a} \mathrm{series} \mathrm{of} \mathrm{voltage}$ regulators. The robot also integrates an $\mathrm{RF}$ module that allows for wireless data exchange with an external PC.

\section{Experimental setup}

The prototype was tested inside a sandbox with dimensions $165 \mathrm{~cm} \times 165 \mathrm{~cm} \times 15 \mathrm{~cm}$ uniformly filled with about $40 \mathrm{~mm}$ of fine seashore sand (mean particle diameter: $0.6 \mathrm{~mm}$ ). The position and orientation of the robot were tracked with a high-definition camera fixed above the experimental area, via computer vision methods, using (a)

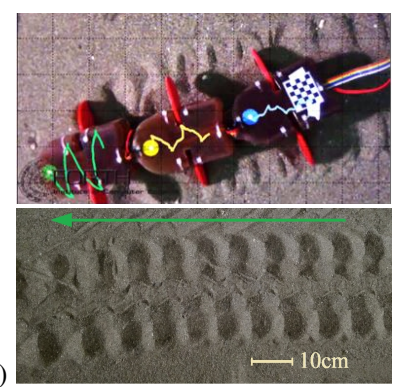

polychaete mode

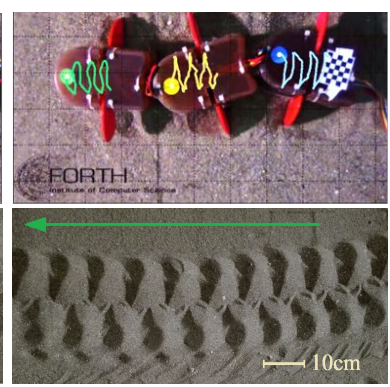

centipede mode
Fig. 13: Experimental results: (a) Trajectories for pedundulatory locomotion of the Nereis prototype with compliant body and joints. A colored marker is used to capture each segment's motion (green: head, yellow: middle, blue: tail; $f=1 \mathrm{~Hz}, A=40^{\circ}, \psi=0^{\circ}$ ). (b) Sand trails from the compliant-body robot with the rigid joints.

a checkerboard marker of known size, placed on the tail segment. In addition, the robot trajectories were recorded and reconstructed by tracking colored markers, mounted on top of each segment: green for the first (head), yellow for the second, and blue for the third (tail) segment (Fig. 13a).

\section{EXPERIMENTAL RESULTS}

In this Section, we present experimental results with the 3 -segment Nereis prototype on sand ${ }^{1}$, performed with the robot powered from an external supply.

Frictional force: To measure the frictional force between the robot's body segments and sand, the experimental setup of Fig. 11a was used, employing a high-precision digital dynamometer (Alluris FMI-210A5). A series of measurements was performed for specific displacement velocities of the dynamometer's base, namely for 5 and $8 \mathrm{~mm} / \mathrm{sec}$. For one segment of the prototype, measurements are taken in both directions, to estimate the tangential and normal frictional force. The friction coefficient was then calculated as $\mu=F / m g$, where $F$ is the measured force, $m$ is the mass of the robot segment, and $g$ is the gravitational constant. The experimental results are shown in Fig. 11b. It is noted that the accumulation of sand in front of the segment affects significantly the measured frictional forces, in particular its normal component. From these measurements, the normal and tangential friction coefficients were estimated as $\mu_{N}=$ 0.5 and $\mu_{T}=0.44$, so that $\mu_{N} / \mu_{T} \approx 1.14$.

Propulsive force: Measurements of the propulsive force generated by the robot in the axial direction, for locomotion on sand, were performed with the experimental setup of Fig. 12a, which also employs the digital dynamometer. Fig. $12 \mathrm{~b}$ presents the average of the peak propulsive force as a function of the amplitude $A$, for the polychaete mode. The generated force, which can be seen to increase with $A$, is higher when rigid body joints are used.

Locomotion velocity: We conducted a series of experiments for pedundulatory locomotion on flat sand, using the experimental setup presented in Section V-D. Indicative trajectories and sand trails for the polychaete and centipede modes are

\footnotetext{
${ }^{1}$ A supplementary video with footage from these experiments can be downloaded from http://tinyurl.com/MED16-forth.
} 


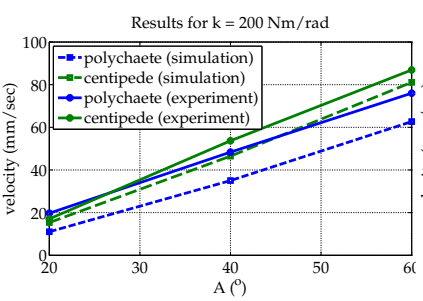

(a) Rigid joints

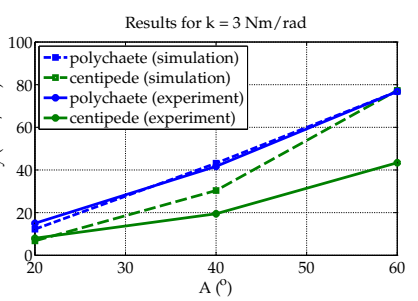

(b) Compliant joints
Fig. 14: Velocities attained with the Nereis robotic prototype (solid lines), shown along with corresponding simulation results (dashed lines), for (a) rigid and (b) compliant body joints.

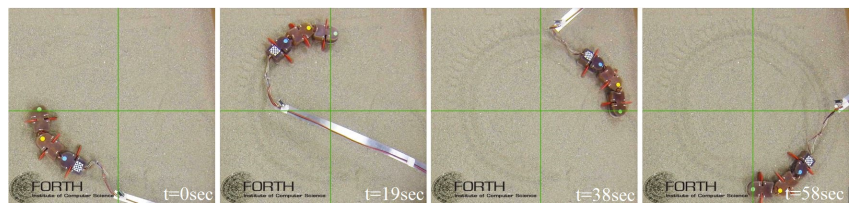

Fig. 15: Experimental results: Turning clockwise in polychaete mode for 75 periods for a complete circular motion (19 periods between snapshots, $f=1 \mathrm{~Hz}, A=35^{\circ}, \psi=25^{\circ}$ ).

shown in Fig. 13. Fig. 14 shows the average velocities attained by the Nereis prototype moving on sand, along with corresponding simulation results, for rigid (modeled with $k=200$ ) and compliant (modeled with $k=3$ ) joints. The experimental results indicate that joint compliance appears to have little effect on the performance of the robot under the polychaete mode. By contrast, the velocities attained in centipede mode are considerably reduced with the compliant joints, compared to the use of rigid joints. However, as noted below, joint compliance was found to increase the adaptation of the robot to its environment, for both modes.

Regarding the predictions of our computational tools, these can be seen to match reasonably well the experimental data. Discrepancies observed may be attributed to phenomena not accounted for in our models (e.g., torsion between the segments, sand accumulation and other phenomena due to the granular nature of the substrate), as well as errors in the specification of the model parameters, such as the friction and stiffness coefficients.

Turning: The most effective turns were performed using a non-zero offset value $\psi$ for the body wave, with the parapodia operating in their normal manner. Other turning strategies (e.g., unilateral parapodial activation with $\psi=0$ ) were less effective. Fig. 15 presents indicative snapshots from these tests, for a complete circle.

Adaptability to unstructured terrains: We investigated the effect of compliance on the adaptability of the system in unstructured, three-dimensional granular formations. Fig. 16a shows a comparison of the compliant-body robot equipped with rigid or compliant joints. In the first case, the robot lost contact with the substrate, some segments were wiggling in the air, and propulsive performance was reduced. Fig. 16b shows how the compliant joints are able to accommodate significant stretching and torsion.

Locomotion on unstructured terrains was further studied by creating various types of protrusions and depressions in the granular substrate. The possibility to traverse these terrains with only the forward gait, i.e. without explicitly

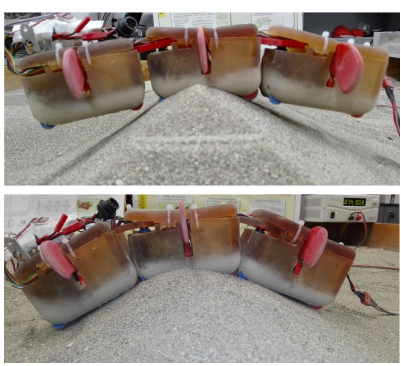

(a)

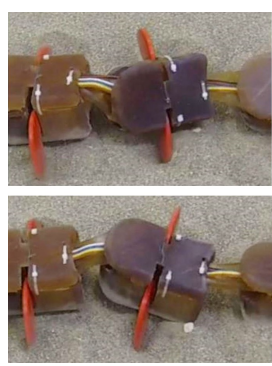

(b)
Fig. 16: Experimental results: (a) Nereis on a sand hill: comparison of the robot with (up) rigid and (down) compliant joints. (b) Segment torsion accommodated by the compliant joints.

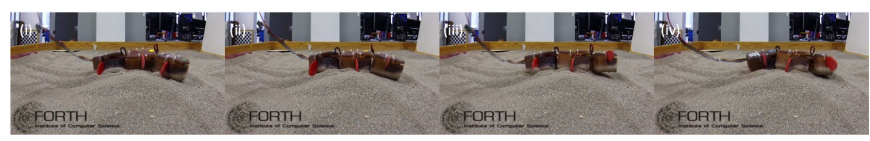

Fig. 17: Experimental results: The Nereis robot with compliant joints traverses a sand hill, in polychaete mode (constant time between snapshots for 1 period, $f=1 \mathrm{~Hz}, \psi=0^{\circ}, A=60^{\circ}$ ).

steering the robot, but merely by the passive adaptation of its shape to the environments features, has been examined. Fig. 17 shows forward propulsion over a sand hill, which is traversed by the compliant-joint robot without difficulty, due to its adaptation to terrain elevation changes; the robot with the rigid joints would instead tend to skirt the hill. Additional tests involved the robot overcoming slopes of different inclinations, as well as following three variants (wide-, medium- and narrow-angled) of a $\Lambda$-shaped trench (see Fig. 18). Our findings, related to the ability of the robot to successfully complete these tasks, using either rigid or compliant joints, are summarized in Table II. The $\Lambda$-shaped trench angles, indicated in Table II, refer to the angle formed by the initial and final portions of the hairpin-like trench. Finally, in Fig. 19, the robot with compliant joints follows a curved $S$-trench, while, again, only employing the forward gait in polychaete mode, by passively adapting its shape to the trench contours. The robot segments can be seen to twist and stretch significantly with respect to each other. The above results highlight the improved terrain traversal capability afforded by joint compliance.

\section{DISCUSSION \& CONCLUSIONS}

In this paper, we present a new pedundulatory robotic prototype featuring a compliant body and compliant joints. The use of parapodia in this system significantly increases the speed and efficiency of the system, compared to pure undulations, in support of our previous studies [18], where the focus was on rigid body and joints. Computational tools, developed in the SIMUUN simulation environment, are employed for modeling compliance in robotic pedundulatory locomotion. These, along with our new experimental

TABLE II: Terrain traversal performance (polychaete mode).

\begin{tabular}{ccc}
\hline Task & Rigid Joints & Compliant Joints \\
\hline Slope crossing (10 $)$ & $\checkmark$ & $\checkmark$ \\
Slope crossing $\left(20^{\circ}\right)$ & $\boldsymbol{x}$ & $\checkmark$ \\
\hline$\Lambda$-trench following (Wide, $100^{\circ}$ ) & $\checkmark$ & $\checkmark$ \\
$\Lambda$-trench following (Medium, $75^{\circ}$ ) & $\boldsymbol{x}$ & $\checkmark$ \\
$\Lambda$-trench following (Narrow, 50 $\left.50^{\circ}\right)$ & $\boldsymbol{x}$ & $\checkmark$ \\
\hline
\end{tabular}




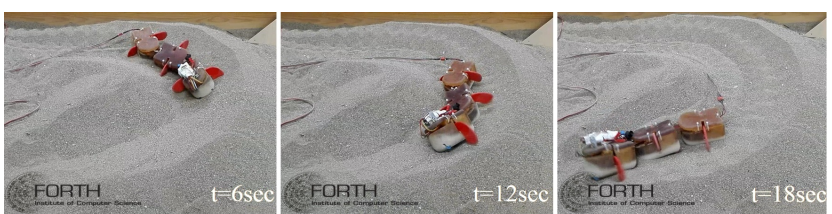

Fig. 18: Experimental results: The robot with compliant joints following a (narrow, $50^{\circ}$ ) $\Lambda$-trench (polychaete mode, $f=1 \mathrm{~Hz}, \psi=$ $\left.0^{\circ}, A=60^{\circ}\right)$.

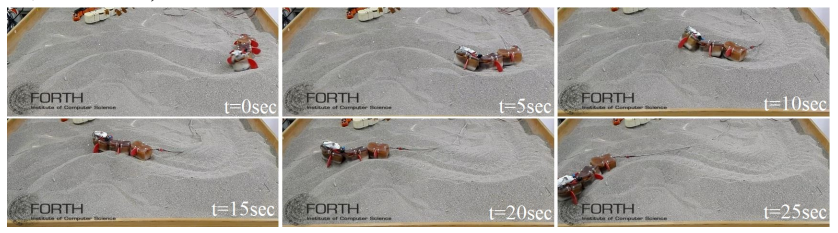

Fig. 19: Experimental results: The robot with compliant joints following an $S$-trench (polychaete mode, $f=1 \mathrm{~Hz}, \psi=0^{\circ}, A=60^{\circ}$ ).

prototype, allow for a number of parametric studies, featuring various levels of compliance in the joints and different biologically-inspired locomotion modes, to investigate their propulsion characteristics in terms of speed, force produced and locomotor efficiency.

The key findings of the present study can be summarized as follows: (i) The use of compliant elements, for interconnecting segments of the body, may improve the ability of the robot to traverse complex, unstructured, and irregular terrain. (ii) Increasing the undulation amplitude of the body, at least in the range under consideration, also increases the average velocity, the propulsive force and the efficiency of the robot. (iii) The use of compliant elements may reduce the average attained velocity and the efficiency, an effect which becomes more significant as the compliance is being increased.

Using both simulations and experiments, our studies highlight the complex and interdependent influence of various parameters of the system (locomotion mode, environmental features, compliance of the coupling elements, etc.) on the propulsion characteristics and efficiency of the robot, and underline the usefulness of the developed computational, design and experimental tools for the analysis and optimization of the design and control of complex robotic locomotor morphologies on sand. Moreover, our experiments appear to indicate that active control of the compliance of the segment interconnection elements also merits further investigation, as it might allow prioritizing, in a more flexible manner, between speed and adaptability, depending on the specific terrain characteristics and mission requirements.

\section{REFERENCES}

[1] S. Hirose, Biologically inspired robots: Snake-like locomotors and manipulators. Oxford University Press, 1993.

[2] J. P. Ostrowski and J. Burdick, "The geometric mechanics of undulatory robotic locomotion," Int. J. Robot. Res., vol. 17, no. 7, pp. 683-701, 1998.

[3] P. S. Krishnaprasad and D. P. Tsakiris, "Oscillations, SE(2)-snakes and motion control: A study of the roller racer," Dyn. Syst., vol. 16, no. 4, pp. 347-397, 2001.

[4] - "G-snakes: Nonholonomic kinematic chains on Lie groups," in Proc. IEEE Conf. Decis. Control (CDC'94), vol. 3, 1994, pp. 29552960.

[5] K. Lipkin, I. Brown, A. Peck, H. Choset, J. Rembisz, P. Gianfortoni, and A. Naaktgeboren, "Differentiable and piecewise differentiable gaits for snake robots," in Proc. IEEE/RSJ Int. Conf. Intell. Robots Syst., 2007, pp. 1864-1869.
[6] M. Saito, M. Fukaya, and T. Iwasaki, "Modeling, analysis, and synthesis of serpentine locomotion with a multilink robotic snake," in IEEE Control Syst. Mag., vol. 22, no. 1, 2002, pp. 64-81.

[7] J. Cortes, S. Martinez, J. P. Ostrowski, and K. A. McIsaac, "Optimal gaits for dynamic robotic locomotion," Int. J. Robot. Res., vol. 20, no. 9, pp. 707-728, 2001.

[8] S. Ma, "Development of a creeping locomotion snake-robot," Int. J. Robot. Automat., vol. 17, no. 4, pp. 146-153, 2002.

[9] P. Liljebäck, K. Pettersen, Ø. Stavdahl, and J. Gravdahl, "A review on modelling, implementation, and control of snake robots," Rob. Auton. Sys., vol. 60, pp. 29-40, 2012

[10] D. Goldman, H. Komsuoglu, and D. Koditschek, "March of the sandbots," IEEE Spectrum, vol. 46, no. 4, pp. 26-31, 2009.

[11] T. Shimada, D. Kadau, T. Shinbrot, and H. Herrmann, "Swimming in granular media," Phys. Rev. E, vol. 80, p. 020301(R), 2009

[12] D. Goldman and D. Hu, "Wiggling through the world," American Scientist, vol. 98, pp. 314-323, 2010.

[13] M. Sfakiotakis, D. P. Tsakiris, and K. Karakasiliotis, "Polychaete-like pedundulatory robotic locomotion," in Proc. IEEE Int. Conf. Robot. Autom. (ICRA'07), 2007, pp. 269-274.

[14] M. Sfakiotakis and D. P. Tsakiris, "Pedundulatory robotic locomotion: Centipede and polychaete modes in unstructured substrates," in Proc. Int. Conf. Rob. Biomim. (ROBIO'08), 2009, pp. 651-658.

[15] G. La Spina, M. Sfakiotakis, D. P. Tsakiris, A. Menciassi, and P. Dario, "Polychaete-like undulatory robotic locomotion in unstructured substrates," IEEE Trans. Robot., vol. 6, pp. 1200-1212, 2007.

[16] M. Sfakiotakis and D. P. Tsakiris, "SIMUUN: A simulation environment for undulatory locomotion," Int. J. Model. Simul., vol. 26, no. 4, pp. 4430-4464, 2006.

[17] — "A biomimetic centering behavior for undulatory robots," Int. J. Robot. Res., vol. 26, no. 11-12, pp. 1267-1282, 2007.

[18] M. Sfakiotakis and D. P. Tsakiris, "Undulatory and pedundulatory robotic locomotion via direct and retrograde body waves," in Proc. IEEE Int. Conf. Rob. Autom. (ICRA'09), 2009, pp. 3457-3463.

[19] K. Melo, M. Garabini, G. Grioli, M. Catalano, L. Malagia, and A. Bicchi, "Open source vsa-cubebots for rapid soft robot prototyping," in Proc. Robot. Sci. Syst. (RSS'14), 2014, pp. 1-5.

[20] M. Vespignani, K. Melo, S. Bonardi, and A. Ijspeert, "Role of compliance on the locomotion of the reconfigurable modular snake robot," in Proc. IEEE/RSJ Int. Conf. Int. Rob. Syst. (IROS'15), 2015, pp. $2238-2245$

[21] S. H. Turlapati, M. Shah, S. P. Teja, A. Siravuru, S. V. Shah, and M. Krishna, "Stair climbing using a compliant modular robot," in Proc. IEEE/RSJ Int. Conf. Int. Rob. Syst. (IROS'15), 2015, pp. 3332-3339.

[22] X. Dong, M. Raffles, S. C. Guzman, D. Axinte, and J. Kell, "Design and analysis of a family of snake arm robots connected by compliant joints," Mech. Mach. Theory, vol. 77, pp. 73-91, 2014.

[23] H. Yamada, S. Takaoka, and S. Hirose, "A snake-like robot for realworld inspection applications (the design and control of a practical active cord mechanism)," Adv. Robot., vol. 27, pp. 47-60, 2013.

[24] M. Vespignani, E. Senft, S. Bonardi, R. Moeckel, and A. Ijspeert, "An experimental study on the role of compliant elements on the locomotion of the self-reconfigurable modular robots roombots," in Proc. IEEE/RSJ Int. Conf. Int. Rob. Syst. (IROS'13), 2013, pp. 43084313.

[25] D. Rollinson, Y. Bilgen, B. Brown, F. Enner, S. Ford, C. Layton, J. Rembisz, M. Schwerin, A. Willig, P. Velagapudi, and H. Choset, "Design and architecture of a series elastic snake robot," in Proc. IEEE Int. Conf. Rob. Autom. (ICRA'14), 2014, pp. 4630-4636.

[26] D. Rollinson, K. V. Alwala, N. Zevallos, and H. Choset, "Torque control strategies for snake robots," in Proc. IEEE Int. Conf. Rob. Autom. (ICRA'14), 2014, pp. 1093-1099.

[27] M. Yim, K. Roufas, D. Duff, Y. Zhang, C. Eldershaw, and S. Homans, "Modular reconfigurable robots in space applications," Auton. Rob., vol. 14, no. 2-3, pp. 225-237, 2003.

[28] J. Gray, "Annelids," in Animal Locomotion. Weidenfeld \& Nicolson, 1968, pp. 377-410.

[29] R. B. Clark and D. J. Tritton, "Swimming mechanisms in nereidiform polychaetes," J. Zool., vol. 161, pp. 257-271, 1970.

[30] B. Anderson, J. Shultz, and B. Jayne, "Axial kinematics and muscle activity during terrestrial locomotion of the centipede, Scolopendra heros," J. Exp. Biol., vol. 198, no. 5, pp. 1185-1195, 1995. 